\title{
ERRATUM
}

\section{VERY HIGH-ENERGY NEUTRINOS FROM SLOWLY DECAYING, MASSIVE DARK MATTER, AS A SOURCE OF EXPLOSIVE ENERGY FOR GAMMA-RAY BURSTS}

\author{
[Mod. Phys. Lett. A, Vol. 18, No. 7 (2003) pp. 477-489] \\ S. Barshay and G. Kreyerhoff
}

The energy unit "ergs", is printed incorrectly as "egrs" throughout the article.

The publisher is sorry for the error. 